\title{
Psychosocial impact of partial or complete edentulism due to periodontal diseases among rural population of Tumkur district of Karnataka
}

\author{
Pushpalatha G ${ }^{1}$, Subbaiah $\mathrm{SK}^{2}$, Bose $\mathrm{A}^{3}$ and Joshipura $\mathrm{V}^{4 *}$ \\ ${ }^{1}$ Department of Periodontics, Sri Siddhartha Dental College and Hospital, India \\ ${ }^{2}$ Department of Periodontics, The Oxford Dental College and Hospital, India \\ ${ }^{3}$ Department of Periodontics, Rajiv Gandhi College of Dental Sciences, India \\ ${ }^{4}$ Intedent Healthcare Private Limited, India
}

\begin{abstract}
Introduction: Periodontal diseases are one of the most prevalent oral diseases affecting more than 50\% of Indian population. Untreated periodontitis is a major cause of tooth loss. In rural population of Tumkur (Karnataka), people live in closely knit large joint families. Partial edentulism at early age could affect marriages, performance at work, criticism from friends and family members and embarrassment at social gatherings.

Objective: We started a cross-sectional study, for assessing the awareness about periodontal diseases and the psychosocial consequences of tooth loss due to periodontal diseases in rural population who visited Sri Siddhartha Dental College.

Methodology: The study involved completion of a predesigned structured questionnaire formatted according psycho-social structure of Indian traditions and culture. It included multiple option questions to be filled up by patients. We screened 799 patients in 18-65 age groups.

Results: The awareness about aggressive periodontitis was less, overall awareness was higher. $88 \%$ patients felt; they would have prevented the disease if there was access to economical dental care. $68 \%$ patients rated their oral health as bad, $59 \%$ did not want to marry partially edentulous person. $83 \%$ experienced criticism, $72 \%$ felt lower level of confidence and $77 \%$ felt quality of life being affected due to partial edentulism.

Conclusion: Partial edentulism due to periodontal disease, especially in young patients, has high psychosocial impact on quality of life. Due to limited replacement options in these poor patients, prevention would give higher quality of life.
\end{abstract}

\section{Introduction}

Periodontal diseases are the most common chronic oral diseases resulting in greatest single cause of tooth loss. Studies have shown high incidence and prevalence of periodontal diseases in India at $57 \%, 67.7 \%, 89.6 \%, 79.9 \%$ in the age groups of $12,15,35-44,65-74$ respectively [1]. The impact of physical, social and psychological influences on the quality of life due to periodontal disease induced tooth loss in the patients, has received much less attention in India $[2,3]$. Dentistry has typically focused on assessments of oral health relevant to the therapists rather than the patient's experience of the disease [4]. Patients' assessments are especially important in periodontal diseases in which their concerns may differ from the traditional clinical endpoints [4]. Over the last decade, patient-centred evaluation tools have been developed and validated to assess patients' subjective oral health in terms of how it affects their daily activities, inter-personal relationships and psychological well-being [2,4]. Many of these tools measure what is termed "Oral health-related quality of life" (OHQoL), which has been defined as "the extent to which oral disorders affect functioning and psycho-social well-being" [4]. In addition to being important outcomes in their own right, the psychosocial experience of oral conditions also plays a role in whether patients seek treatment and follow advice and could thus influence the planning and process of treatment [4].
Measures of oral health-related quality of life (OHRQoL) are increasingly being used in descriptive population-based research as a means of capturing nonclinical aspects of oral health that patients deem most relevant to their overall health and well-being [2,3].

Periodontal disease has been reported to be asymptomatic during most of its course, with clinical signs, such as periodontal pockets, being generally painless $[5,6]$. In its more advanced stages, periodontal disease can be associated with signs and symptoms that are readily perceivable by individuals, such as tooth mobility, pain, difficulties in eating, aesthetically compromised loss of anterior interproximal papillae, or discomfort $[7,8]$.

In rural population of Tumkur, people live in closely knit large joint families. High prevalence of periodontal diseases, lack of proper dental care facilities and lack of awareness result in partial edentulism due to periodontal disease. Thus, the aim of this study was to assess the

${ }^{\star}$ Correspondence to: Vaibhavi Joshipura, Intedent Healthcare Private Limited, India, Tel: 9880105198; E-mail: vaibhavi_joshipura@yahoo.co.in

Key words: psychosocial impact, partial edentulism, periodontal disease, awareness Received: April 10, 2019; Accepted: April 25, 2019; Published: April 29, 2019 
psychosocial impact of partial or complete edentulism due to periodontal diseases among rural population of Tumkur district of Karnataka.

\section{Aims and objectives}

- To assess knowledge and awareness about the periodontal diseases, among rural populations in Tumkur district.

- To investigate, the effects of losing anterior teeth due to periodontal diseases and its consequences on social and psychological aspects of patients' life.

\section{Material and methods}

\section{Study design}

This study was designed as a prospective cross-sectional study to assess psycho-social consequences of losing anterior teeth due to periodontal disease. The study was approved by institutional ethical committee. All patients received detailed description of the study protocol and a written informed consent was taken from them.

\section{Source of data}

Patients reporting to Sri Siddhartha dental college, Tumkur, Karnataka, during November 2009 to May 2012 were screened for loss of anterior teeth due to periodontal disease. The statistical analysis was done by December 2012.

\section{Inclusion criteria}

1. Loss of at least one anterior tooth due to periodontal disease.

2. Patients in the age group of 15 to 65 years.

\section{Exclusion criteria}

1. Anterior teeth lost due to caries, trauma, congenitally missing teeth or due to any other causes other than periodontal diseases.

We screened 799 patients in 15-65 age groups. Two examiners were involved in the study. One was responsible for structuring the questionnaire, its conversion to kannada language, and formatting consent form. The other examiner was responsible for assigning questionnaire to the patients and receiving filled questionnaires from patients.

\section{Study protocol}

The patients were subjected to a detailed case history along with the age and educational status to confirm their eligibility criteria. The study involved completion of a predesigned structured questionnaire. It included multiple option questions to be filled up by patients. The questionnaire was based on OHQoL-UK, along with few more questions addressing the social issues in this region.

The primary objective of our study was to assess social and psychological aspects of edentulism rather than function and disability. We had to make changes in the variables according to the psychosocial aspects in the rural population of Tumkur. The rural population in Tumkur has no access to regular dental care. The awareness about periodontal diseases and its consequences was never determined in this population. The level of education was also minimal. People live in large joint families where the head of the family would take major decision of the life of an individual.

Considering these social and cultural aspects, OHQoL questionnaire was modified into 4 sections containing total 22 questions.
1. Awareness about periodontal diseases: This section consisted questions on periodontal health-systemic health correlation, periodontal health-oral health correlation, types of periodontal diseases, age and periodontal diseases, genetics basis of periodontal diseases and prevention of periodontal diseases.

2. Knowledge about consequences of periodontal diseases covered consanguinity, periodontal diseases and halitosis, bleeding gums and pus discharge, diastema which did not exist before, pain in gums and teeth and mobility of the teeth.

3. Social aspects of partial/complete edentulism had questions on loss of front teeth, mobility of teeth, flaring of anterior teeth.

4. Psychological aspects of partial/complete edentulism addressed the issues of criticism, confidence, spouse selection, marriage with edentulous individual, prevention if known, quality of life and rate oral health.

A 3-point likert scale was used to answer the questionnaire i.e. yes, no or don't know, to make it simple and easier for the patients to understand and answer and to avoid statistical bias.

The questionnaire was structured in English. One staff member was authorized to translate it to Kannada, which was verified by team of two staff members from other departments.

One staff from language department was then asked to translate questionnaires in Kannada to English again in order to cross verify the Kannada questionnaires.

All the participants were given a questionnaire in Kannada to be filled up.

\section{Statistical analysis}

The results were assessed according to the sections in the questionnaire. Evaluation was also made according to age, sex and educational qualification.

After data collection, 799 questionnaires were statistically analysed. Basic analysis of frequencies of the various responses was performed. Outcome variables were the awareness (No 1 to 6), knowledge (Q no 7 to12), social aspects (Qno.13 to 15), psychological aspects (Q. no16 to 22), age, gender and educational status.

The explanatory variables were age, gender and education recoded for meaningful statistical analyses into age groups 15 to 24 years, 25 to 34 years, 35 to 44 years, 45 to 54 years, and 55 to 65 years.

In all instances when participants failed to respond to certain questions, analyses were based only on the number of valid responses. This means that figures in the results did not always sum up to 799. Explanatory footnotes were inserted in such instances.

\section{Results}

The results were assessed according to the sections in the questionnaire. Evaluation was also made according to age, sex and educational qualification.

As the age group table suggests maximum number of patients volunteered were in the age group of 15-25. Number of male and female participants were $60 \%$ and $40 \%$. The educational status suggested $10 \%$ less than $10^{\text {th }}$ grade, $35 \%$ up to $10^{\text {th }}$ grade, $18 \%$ till high school and $37 \%$ had bachelor's degree. 
In the awareness section we tried to evaluate whether patients were aware about onset, aetiology as well as connection of periodontal health to oral as well as systemic health. (Table 1 and Graph 1) Around $84 \%$ patients were not aware about periodontal diseases happening in young age or could be aggressive. Patients who did not know that the periodontal disease could be chronic or fast progressing was around $43 \%$. About $46 \%$ of the subjects believed that periodontal disease had no genetic component as major etiological factor and 32\% did not know about genetic aetiology. The percentage of patients who thought that periodontal disease could not be prevented by maintaining oral hygiene was $44 \%$, whereas $54 \%$ thought that they are preventable. About $94 \%$ of the patients thought that keeping gums healthy will keep them healthy and $89 \%$ thought periodontal and oral health was related.

The second section consisted of questions related to consequences and signs and symptoms of periodontal disease (Table 2 and Graph 2). Around $43 \%$ of the patients believed that the disease is not seen in families. Around $81 \%$ connected halitosis with presence of periodontal disease, $85 \%$ thought periodontal disease resulted in bleeding gums and pus discharge. The percentage of patients who thought that diastema, pain and mobility were signs of gum disease was $73 \%, 86 \%$, and $83 \%$ respectively.

Third section related to social aspect assessed loss of anterior teeth, mobility of teeth and pathologic migration of teeth hampering their social engagements. Around $84 \%$ of the patients were not bothered if their anterior teeth had flared, protruded or pathologically migrated.

Table 1. Awareness about periodontal diseases

\begin{tabular}{|l|c|c|c|}
\hline 1 Awareness questions & Yes(A) & No(B) & $\begin{array}{c}\text { Don't. } \\
\text { Know(C) }\end{array}$ \\
\hline Periodontal-systemic health & $94 \%$ & $2 \%$ & $4 \%$ \\
\hline Periodontal-oral health & $89 \%$ & $5 \%$ & $6 \%$ \\
\hline Types of periodontal diseases & $51 \%$ & $5 \%$ & $43 \%$ \\
\hline Age and periodontal diseases & $8 \%$ & $8 \%$ & $84 \%$ \\
\hline Genetics and periodontal diseases & $23 \%$ & $46 \%$ & $32 \%$ \\
\hline Prevention of periodontal diseases & $54 \%$ & $44 \%$ & $2 \%$ \\
\hline
\end{tabular}

\section{Graph 1-Awareness about periodontal disease}

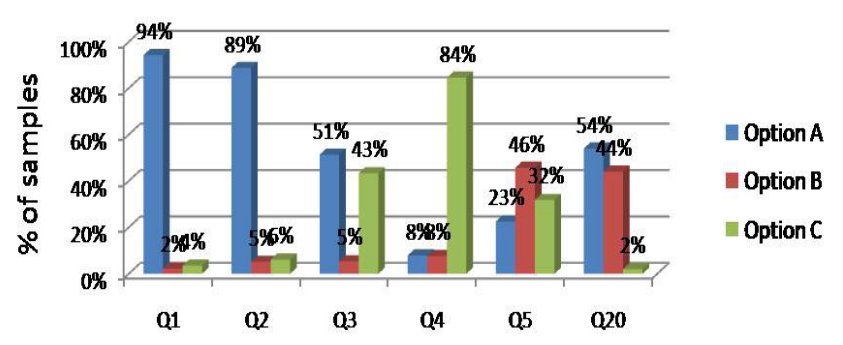

Table 2. Knowledge about consequences of periodontal diseases.

\begin{tabular}{|l|c|c|c|}
\hline $\begin{array}{l}\text { Knowledge about } \\
\text { periodontal } \\
\text { diseases }\end{array}$ & Yes(A) & No(B) & Don't know(C) \\
\hline Familial aggregation & $25 \%$ & $43 \%$ & $32 \%$ \\
\hline halitosis & $81 \%$ & $9 \%$ & $10 \%$ \\
\hline $\begin{array}{l}\text { Bleeding gums and } \\
\text { pus discharge }\end{array}$ & $85 \%$ & $4 \%$ & $11 \%$ \\
\hline Diastema & $73 \%$ & $10 \%$ & $17 \%$ \\
\hline Pain & $86 \%$ & $5 \%$ & $9 \%$ \\
\hline Mobility & $83 \%$ & $7 \%$ & $10 \%$ \\
\hline
\end{tabular}

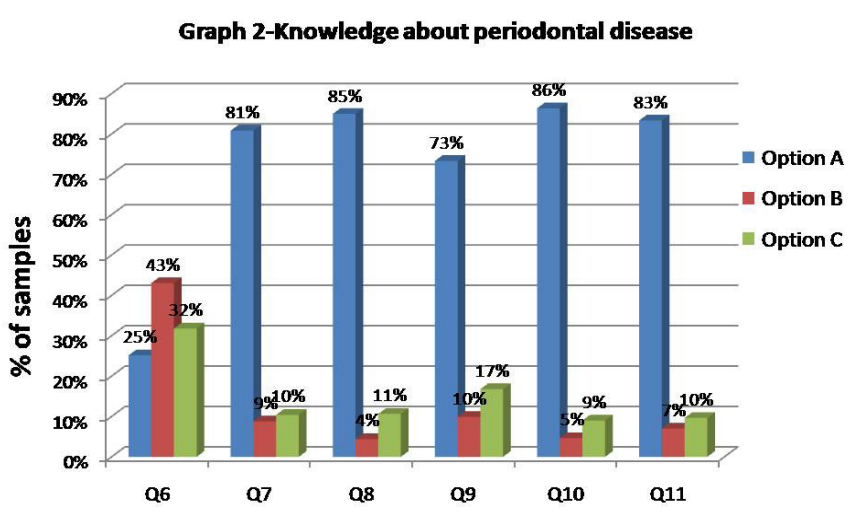

However, $86 \%$ and $89 \%$ felt loss of anterior teeth and mobile anterior teeth were socially unacceptable to them.

Interestingly, psychological concerns were profound with this rural population with no access to dental care. About $88 \%$ of the patients said if they knew about prevention, they would have opted for it, but then they did not know. The percentage of patients who faced criticism by family, friends and others was $83 \%$. Around $72 \%$ of the patients felt they had lack of confidence because of edentulism. For $56 \%$ of the patients, anterior edentulism was an important aspect for spouse selection but on the other hand 59\% said they would not get married to a person with anterior edentulism. It would be logical to state that majority of anterior edentulous areas in these patients are not replaced prosthetically due to lack of economical and viable options. Some of them had removable partial dentures, but they were not comfortable with it. Around $67 \%$ of patients rated their oral health as bad and $77 \%$ said that anterior edentulism affected their quality of life.

\section{Discussion}

A shift towards a focus on quality of life aspect and subjective needs, rather than morphological deficits, can be seen in both medicine and dentistry [6]. In dentistry, mostly a 'need approach' has been used, where the importance of all determinants of quality of life are equal for all individuals. In medicine, a 'want approach' has been introduced [7], also called: 'the human judgement analysis' [8]. According to this approach, quality of life can only be affected by factors important to the individual, such as lifestyle, previous experience, ambitions, hopes and dreams [7]. Health is a state of complete physical, mental, and social well-being and not merely an absence of disease. WHO (1948) Oral Health-Related Quality of Life (OHRQoL) is defined by individual assessment of several oral health dimensions including physical dental function, tooth pain, psychological discomfort, and social impacts-all of which affect overall well-being [9].

The present study intended to assess knowledge and awareness about the periodontal diseases, among rural populations in Tumkur district of Karnataka, India and also investigated the effects of losing teeth and the psycho-social impact of partial or complete edentulism due to periodontal diseases in the life of patients.

This was a prospective cross-sectional study conducted at Sri Siddhartha dental college, Tumkur, Karnataka, during November 2009 to May 2012. Out of 799 subjects who were assessed by means of questionnaire $60 \%$ (476) were males and $40 \%$ (323) were females. Maximum number of patients volunteered was in the age group of 1525 . Educational status of the population revealed $10 \%$ (80) were $<$ SSLC (less than $10^{\text {th }}$ grade), $35 \%$ (277) had studied till SSLC (up to $10^{\text {th }}$ grade, 
18\% (146) had studied till PUC (high school) and the rest $37 \%$ (276) had finished their bachelor's degree.

The population in rural India frequently succumbs to social stigma because of physical ailments of which dental problems are one. Edentulism due to periodontal diseases is not uncommon in the geriatric population [10]. The present study had a total of 49 subjects in the age group of 55-65 years but surprisingly in this populationbased study the volunteers with edentulism were maximum in the age group of 15-25years with male participants having the dominance. This implies that edentulism due to periodontal diseases is more frequent in the middle age group compared to older age group. This is in contrast to studies which proved that edentulism is common in geriatric population [10]. Educational qualification is a clear indicator of health awareness. Higher the qualification better is the awareness [11]. This is clearly reflected in our results as $37 \%$ of population who were graduates responded positively for question pertaining to awareness of periodontal diseases and their impact on general health. Periodontal diseases are quiescent by nature. Hence, even before the patient is aware the disease is in an intractable state [12]. As a consequence of this, patient awareness of periodontal disease is less [13].

In the awareness section we tried to evaluate whether patients were aware about onset, aetiology as well as connection of periodontal health to oral as well as systemic health (Table 3 and Graph 3). Echoing this fact, $84 \%$ of subjects in our study were not aware of the periodontal diseases happening in young age and $43 \%$ of subjects had the faintest idea about the progress of periodontal disease. In contrast, $44 \%$ of subjects in the study did not believe that periodontal diseases can be prevented by maintaining oral hygiene and $54 \%$ of subjects believed it could be.

Concomitant to it, $94 \%$ of the respondents thought that keeping gums healthy will keep them healthy and $89 \%$ believed that periodontal and oral health were inter-related. This is in parallelism with studies which proved a better awareness of Indian rural population towards periodontal health [14].

The second section consisted of questions related to consequences and signs and symptoms of periodontal disease (Table 4 and Graph 4). The common symptoms of periodontal diseases are halitosis, bleeding gums, pus discharge and mobility of teeth. Studies have also proven a genetic link for periodontal diseases [15] but whether subjects believed

Table 3. Social aspects of edentulism

\begin{tabular}{|l|c|c|c|}
\hline Social aspects of partial edentulism & Yes(A) & No(B) & Don't know (C) \\
\hline Loss of front teeth & $86 \%$ & $8 \%$ & $5 \%$ \\
\hline Mobility of teeth & $89 \%$ & $8 \%$ & $3 \%$ \\
\hline Flaring of anterior teeth & $13 \%$ & $84 \%$ & $4 \%$ \\
\hline
\end{tabular}

\section{Graph 3-Social Aspects of Edentulism}

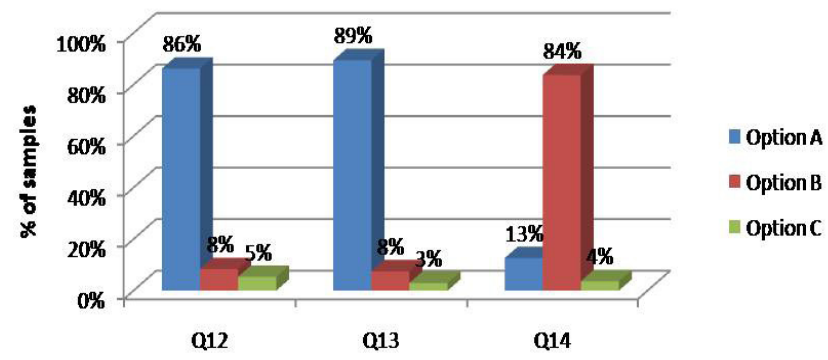

Table 4. Psychological aspects of edentulism

\begin{tabular}{|l|l|l|l|}
\hline Psychological aspects & Yes(A) & No(B) & Don't know(C) \\
\hline Criticism & $83 \%$ & $12 \%$ & $6 \%$ \\
\hline Confidence & $72 \%$ & $15 \%$ & $13 \%$ \\
\hline Spouse selection & $56 \%$ & $37 \%$ & $7 \%$ \\
\hline Marriage with edentulous & $39 \%$ & $59 \%$ & $2 \%$ \\
\hline Prevent if knew & $88 \%$ & $12 \%$ & $1 \%$ \\
\hline Quality of life & $77 \%$ & $23 \%$ & $0 \%$ \\
\hline Rate oral health & $32 \%$ & $67 \%$ & $1 \%$ \\
\hline
\end{tabular}

Graph 4- Psychological Aspects of Edentulism

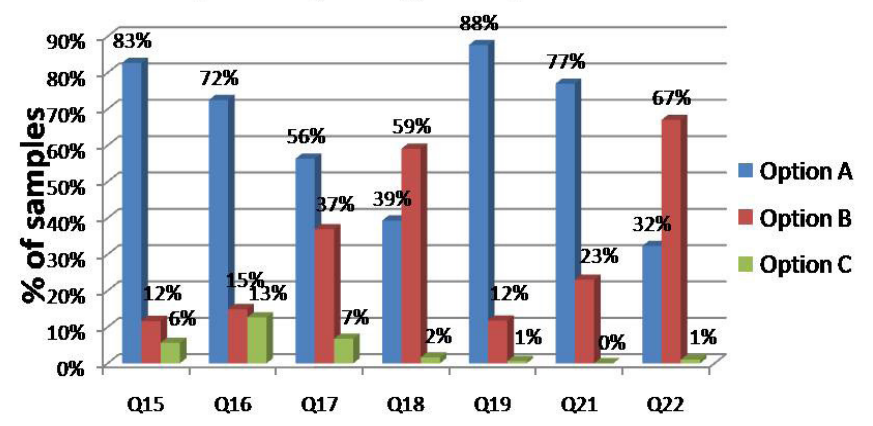

that these symptoms are indicative of periodontal disease and whether genetics play an important role was evaluated in this section. Majority of the respondents above $80 \%$ believed halitosis, bleeding gums, pain and mobility of teeth to be a consequence of periodontal disease where as $73 \%$ of the subjects attributed diastema to be a symptom of gum disease.

Anti-parallel to the genetic contribution to periodontal disease, $43 \%$ of the subjects in our study believed that periodontal disease is not seen in families. The percentage of respondents who were indecisive about symptoms of periodontal disease represented the group who were unaware about knowledge and consequences of periodontal diseases.

A smile adds to the charm of the face and better aesthetics contributes to higher social acceptance [16] Therefore, the third section which was related to the social aspect assessed loss of anterior teeth, mobility of teeth and pathologic migration of teeth hampering their social engagements. All of these are fall outs of periodontal disease which depreciate the aesthetics, thus leading to lower social acceptance. In spite of this cliché, $84 \%$ of the respondents in our study were indifferent towards lack of aesthetics due to periodontal diseases. However, $89 \%$ of our respondents were of the belief that loss and mobility of anterior teeth were socially unacceptable.

The psychological dimensions of oral health comprise of awareness, availability, accessibility, affordability and acceptance in the social circle (REF). $88 \%$ of the subjects accepted that it was their lack of awareness that prevented them from approaching dental care. Better aesthetics is a direct indicator of improved social acceptance [17]. Echoing this, $83 \%$ of the subjects faced criticism from family and friends and $72 \%$ exhibited lack of confidence due to edentulism. Social acceptance in terms of getting married is regulated by edentulism. Around $59 \%$ of the subjects in our study said that they won't get married to a person with anterior edentulous area.

Complying with the economic dimensions of oral health, $59 \%$ of the respondents could not afford replacement of anterior teeth due to lack of financial availability; or even if they could afford the dental care it was with a compromise in terms of comfort with a RPD. In complete 
essence, after being sensitized to a comprehensive oral health awareness by means of a questionnaire, $67 \%$ of the respondents, rated their oral health as bad and $77 \%$ were of the belief that anterior edentulism affected their quality of life which is what is indicative of psychosocial impact of partial or complete edentulism. Oral health quality deserves to be promoted in the national oral health plan to meet the needs of the population and achieve the optimal benefits from available resources. OHRQoL can measure the effectiveness of dental public health programmes, assessing the oral health needs of populations.

\section{Summary and conclusion}

This epidemiological study points to the fact that there is an increased prevalence of severe periodontal disease in the young rural population of Tumkur, Karnataka. One of the major reasons could be lack of awareness as well as regular dental care facility in the area. Genetic contribution due to consanguinity cannot be ruled out as an important etiologic factor in this population (our genetic study reference), environmental factors can be controlled if they are incorporated in health care policies.

The psychosocial analysis of the population with partial edentulism reveals that most of the participants felt criticism, embarrassment, and low confidence level due to loss of front teeth to periodontal disease and wished they had better replacement options. They admitted their quality of life was getting affected by it.

The authors would also like to conclude that there is a need to address the issue of affordable replacement options and stress on prevention of periodontal disease by access to regular dental care for better quality of life in Tumkur Population.

\section{References}

1. Shaju JP, Zade RM, Das M (2011) Prevalence of periodontitis in the Indian population: A literature review. J Indian Soc Periodontol 15: 29-34. [Crossref]

2. Slade GD (2002) Assessment of oral health-related quality of life. In: Inglehart MR, Bagramian RA editors. Oral health-related quality of life. Carol Stream, IL: Quintessence Publishing Co; 29-45.
3. Lawrence HP, Thomson WM, Broadbent JM, Poulton R (2008) Oral health-related quality of life in a birth cohort of 32-year olds. Community Dent Oral Epidemiol 36: 305-316. [Crossref]

4. Jowett AK, Orr MT, Rawlinson A, Robinson PG (2009) Psychosocial impact of periodontal disease and its treatment with 24 -h root surface debridement. $J$ Clin Periodontol 36: 413-418. [Crossref]

5. Cunha-Cruz J, Hujoel PP, Kressin NR (2007) Oral health-related quality of life of periodontal patients. J Periodontal Res 42: 169-176. [Crossref]

6. Novak MJ, Novak KF (2002) Chronic Periodontitis. Carranza's Clinical Periodontology, 10th edition. 494-499.

7. Blicher B, Joshipura K, Eke P (2005) Validation of self-reported periodontal disease: a systematic review. J Dent Res 84: 881-890. [Croosref]

8. Needleman I, McGrath C, Floyd P, Biddle A (2004) Impact of oral health on the life quality of periodontal patients. J Clin Periodontol 31: 454-457. [Crossref]

9. Yiengprugsawan V, Somkotra T, Seubsman SA, Sleigh AC, Thai Cohort Study Team (2011) Oral Health-Related Quality of Life among a large national cohort of 87,134 Thai adults. Health Qual Life Outcomes 9: 42. [Crossref]

10. Polzer I, Schimmel M, Müller F, Biffar R (2010) Edentulism as part of the general health problems of elderly adults. Int Dent J 60: 143-155. [Crossref]

11. Gambhir RS, Sohi RK, Nanda T, Sawhney GS, Setia S (2013) Impact of School Based Oral Health Education Programmes in India: A Systematic Review. J Clin Diagn Res 7: 3107-3110. [Crossref]

12. Brady WF (1984) Periodontal disease awareness. J Am Dent Assoc 109: 706-710. [Crossref]

13. Croxson LJ (1998) Practical periodontics. Awareness of periodontal disease in the patient. Int Dent J 48: 256-260. [Crossref]

14. Grewal Y, Datta R, Singh K, Singh G, Singh S, et al. (2014) Prevalence of Periodontal Disease in the Rural Population of Punjab. J Pharm Biomed Sci 4: 532-535.

15. Taba M Jr, Souza SL, Mariguela VC (2012) Periodontal disease: a genetic perspective. Braz Oral Res 26 1: 32-38. [Crossref]

16. Klages U, Bruckner A, Zentner A (2004) Dental aesthetics, self-awareness, and oral health-related quality of life in young adults. Eur J Orthod 26: 507-514. [Crossref]

17. Tin-Oo MM, Saddki N, Hassan N (2011) Factors influencing patient satisfaction with dental appearance and treatments they desire to improve aesthetics. BMC Oral Health 11: 1-8. [Crossref]

Copyright: (C2019 Pushpalatha G. This is an open-access article distributed under the terms of the Creative Commons Attribution License, which permits unrestricted use, distribution, and reproduction in any medium, provided the original author and source are credited. 